\title{
Concentrations of Estradiol, Progesterone and Testosterone in Serum and Cerebrospinal Fluid of Patients with Aneurysmal Subarachnoid Hemorrhage Correlate Weakly with Transcranial Doppler Flow Velocities
}

Jan Martin ( $\nabla$ j.martin@tum.de)

Technische Universitat Munchen Fakultat fur Medizin https://orcid.org/0000-0001-8838-0834

Eva Plank

Technische Universitat Munchen Fakultat fur Medizin

Bernhard Ulm

Technische Universitat Munchen Fakultat fur Medizin

Jens Gempt

Technische Universitat Munchen Fakultat fur Medizin

Maria Wostrack

Technische Universitat Munchen Fakultat fur Medizin

Bettina Jungwirth

Universitatsklinikum Ulm

Simone Maria Kagerbauer

Technische Universitat Munchen Fakultat fur Medizin

\section{Research article}

Keywords: Aneurysmal subarachnoid hemorrhage, vasospasm, estradiol, progesterone, testosterone, cerebrospinal fluid, blood, human

Posted Date: September 22nd, 2020

DOI: https://doi.org/10.21203/rs.3.rs-63644/v1

License: (c) (1) This work is licensed under a Creative Commons Attribution 4.0 International License. Read Full License

Version of Record: A version of this preprint was published at BMC Neuroscience on April 23rd, 2021. See the published version at https://doi.org/10.1186/s12868-021-00634-3. 


\section{Abstract}

\section{Background:}

The implication of the steroids estradiol, progesterone and testosterone in cerebral vasospasm after aneurysmal subarachnoid hemorrhage (aSAH) has not been comprehensively assessed. In rodents, studies suggested beneficial effects of steroids on cerebral vasospasm after experimental SAH. Studies in humans are warranted, however, a general dilemma of human studies on neuroactive substances is that the brain is not directly accessible and that concentrations in the periphery may not adequately parallel concentrations in the central compartments. In the present study, concentrations of estradiol, progesterone and testosterone in serum and cerebrospinal fluid (CSF) of patients with aSAH were determined. Blood flow velocities in cerebral arteries were measured by transcranial Doppler sonography (TCD). The aim of this study was to evaluate the correlations between the cerebral blood flow velocities and levels of estradiol, progesterone and testosterone in CSF and serum.

\section{Results:}

Samples of serum and CSF of 42 patients with aSAH were collected concomitantly daily or every other day via the arterial line and the external ventricular drainage for two weeks after the hemorrhage. Blood flow velocities in the cerebral arteries were determined by TCD. Total estradiol, progesterone and testosterone concentrations were measured by electro-chemiluminescence immunoassay. The strength of correlation was assessed by Spearman's rank correlation coefficient. The correlation analysis revealed very weak correlations between cerebral blood flow velocities and concentrations of estradiol, progesterone and testosterone levels in both compartments with correlation coefficients below 0.2 .

\section{Conclusions:}

In humans with aSAH, merely very weak correlations between flow velocities of cerebral arteries and concentrations of estradiol, progesterone and testosterone in serum and CSF were demonstrated. The relevance of steroids on cerebral vasospasm in humans remains unclear although beneficial effects were described in rodent studies. Pathways leading to neurological deterioration linked and unlinked to cerebral vasospasm remain complex and possibly interrelated. Thus, the implication of steroids in cerebral vasospasm or other processes of neurological deterioration warrants further clarification.

\section{Background}

Aneurysmal subarachnoid hemorrhage (aSAH) refers to the spontaneous rupture of an intracranial aneurysm. Despite improvements in microsurgery, interventional neuroradiology and neurocritical care the disease continues to be associated with a high risk of morbidity and mortality. The issue of delayed neurological deterioration after aSAH has long been addressed and remains subjected to studies in order to shed light on a complex system of processes due to cerebral vasospasm and due to processes that seem to be unrelated to cerebral vasospasm $(1,2)$. 
In the development of neurological deterioration due to cerebral vasospasm after aSAH an endothelial damage or inflammatory causes are commonly discussed $(2,3)$. Steroids appear involved in these processes but it is still largely unsettled to what extent the steroids estradiol, progesterone and testosterone have an influence on the development or degree of cerebral vasospasm. In rodents, several studies assessed the potentially beneficial effects of these steroids on cerebral vasospasm after experimental subarachnoid hemorrhage (SAH) $(2,4,5)$. A further knowledge of the implication of estradiol, progesterone and testosterone in differing compartments, such as blood, cerebrospinal fluid (CSF) and brain parenchyma in influencing cerebral vasospasm may help to understand factors underlying its pathogenesis or unveil therapeutic options. Studies in humans are warranted, however, a general dilemma of human studies on neuroactive substances is that the brain is not directly accessible and that concentrations in periphery, e.g. blood, may not adequately parallel concentrations in brain parenchyma. Presuming that steroid levels in the CSF may reflect steroid activity in the brain, this dilemma can be approached but in principle persists. Also, human CSF is not easy to obtain and usually requires invasive procedures such as lumbar puncture or ventricular drainage whereby the latter is often performed in aSAH patients as a therapeutic measure.

In the present study, concentrations of estradiol, progesterone and testosterone in CSF and blood of patients with aSAH were determined. Blood flow velocities in cerebral arteries were measured by transcranial Doppler sonography. The aim of this study was to evaluate the correlations between the blood flow velocities in the cerebral arteries and levels of estradiol, progesterone and testosterone in CSF and serum.

\section{Methods}

The study was approved by the ethics committee of the medical faculty of the Technical University Munich (project number 2410/09). We prospectively studied 42 patients (mean age 57 years, range 20 to 80 years) with aneurysmal SAH (table 1). Written informed consent was provided by all patients or their legal guardians respectively. The patient's clinical condition after the onset of the disease was assessed by the grading scale of Hunt \&Hess (6). The blood flow velocities in the anterior, middle and posterior cerebral arteries were measured by transcranial Doppler sonography (TCD). Cerebral infarction was diagnosed by computer tomography. Patient outcome was assessed on the Glascow Outcome Scale (7) at three months after the ictus.

Samples of CSF and serum were collected concomitantly daily or every other day via the arterial line and the external ventricular drainage during the first two weeks after the hemorrhage. Total estradiol, progesterone and testosterone concentrations in CSF and serum were measured on a Roche Mannheim Cobas e 411 immunoassay analyzer using an electro-chemiluminescence immunoassay (detection limit of $5 \mathrm{pg} / \mathrm{ml}$ for estradiol, detection limit of $0.03 \mathrm{ng} / \mathrm{ml}$ for progesterone, detection limit of $0.025 \mathrm{ng} / \mathrm{ml}$ for testosterone). The TCD measurements were also performed daily or every other day. 
Strength of bivariate monotonous correlation of quantitative data was assessed by Spearman's rank correlation coefficient. Correlations were analyzed between the maximum systolic blood flow velocity determined in a cerebral artery and the concentrations of estradiol, progesterone and testosterone in serum und CSF respectively per measurement day. The calculation of each correlation coefficient was based on 281-286 observations according to the steroid and the compartment investigated. An absolute value of the correlation coefficient of $0.00-0.19$ indicates a very weak correlation, $0.20-0.39$ a weak correlation, $0.40-0.59$ a moderate correlation, $0.60-0.79$ a strong correlation and a value of $0.80-1.0 \mathrm{a}$ very strong correlation.

Group comparisons were performed to evaluate associations between steroid concentrations in the respective compartments and the grading of the Hunt \& Hess scale, the grading of the Glascow Outcome scale and the occurrence of cerebral infarction. The two-group-comparisons of steroid concentrations in serum and CSF and patients with and without cerebral infarction were conducted using the Mann Whitney test. For the multi-group-comparisons (Hunt \& Hess scale grades, Glascow Outcome scale grades) Kruskal-Wallis-testing was used. Hypothesis testing was performed on two-sided $5 \%$ significance levels. Statistical analysis was done by using R software 3.5.0 (R Foundation for Statistical Computing, Austria, Vienna).

\section{Results}

The patient characteristics are presented in table 1. The data on concentrations of estradiol, progesterone and testosterone in serum and CSF as well as the data on the range of the maximum velocities per day measured by transcranial Doppler sonography in a cerebral artery are listed in table 2 . The mean concentrations and standard deviations of estradiol, progesterone and testosterone of the overall cohort are demonstrated in table 3 .

The Spearman's rank correlation coefficients between the serum und CSF concentrations of estradiol, progesterone and testosterone and the transcranial Doppler flow velocities are presented in table 4 . The correlation analysis revealed merely very weak correlations between cerebral flow velocities and concentrations of estradiol, progesterone and testosterone levels in any of the two compartments with correlation coefficients below 0.2 (table 4).

The group comparisons between steroid concentrations in the respective compartments and the grading of the Hunt \& Hess scale, the grading of the Glascow Outcome scale and cerebral infarction revealed no significant differences.

\section{Discussion}

In humans with aSAH, possible interrelations between cerebral vasospasm and the steroids estradiol, progesterone and testosterone in peripheral compartments, e.g. blood, or in central compartments, e.g. $\mathrm{CSF}$, have not yet been comprehensively assessed. Given numerous studies in rodents on steroids, 
predominantly estradiol, and its association with cerebral vasospasm after experimental SAH, the idea that steroids might be involved in processes linked to cerebral vasospasm or functional outcome remains to be investigated in more detail. This investigation analyzed the correlations between transcranial Doppler flow velocities in cerebral arteries and concentrations of estradiol, progesterone and testosterone in serum and CSF of patients with aSAH. The results showed very weak correlations between the transcranial Doppler flow velocities and the steroid concentrations in both compartments.

The neuroprotective influences attributed to estradiol in aSAH comprise vasodilatory effects and general neuroprotective mechanisms. With regard to the vasodilatory effects of estradiol, such as countermanding vasoconstriction or promoting vasodilation, studies focused on nitrogen monoxide (NO), reactive oxygen species (ROS) and endothelins (ET) since endothelial damage and inflammation after aSAH remain discussed as key determinants of cerebral vasospasm. Various nitric oxide synthase (NOS) enzymes are identified, there among endothelial NOS (eNOS), neuronal NOS (nNOS) and inducible NOS (iNOS) (8). Chen et al. demonstrated in studies on ovine endothelial cells that the vasodilatory effects of estradiol are mediated by estradiol's capacity to activate eNOS and that the activation of eNOS was impeded by blocking the estrogen receptor (9). Likewise, an in vitro study by Nevzati et al. in human umbilical and brain endothelial cells treated with estradiol showed a rise in NO and eNOS concentrations presumably due to estradiol mediated pathways (10). Also, Selles et al. showed in endothelial cells of rat aorta that treatment with estradiol and progesterone enhanced NOS activity (11). In a canine SAH model, Khurana et al. found a protective effect of recombinant eNOS against cerebral vasospasm (12). Studies have also addressed the issue of inflammation as a critical factor for vasospasm and evaluated a potentially positive impact of steroids on these processes. SAH may entail formation of oxy-hemoglobin and inflammatory cascades with an increase of ROS thereby decreasing NO levels and thus facilitating cerebral vasoconstriction $(3,13,14)$. Furthermore, Ding et al. discussed that vascular inflammation may enhance iNOS activity resulting in an increased ROS generation hence promoting inflammatory pathways and cerebral vasospasm (2). In this respect Handa et al. found in primates good correlations between angiographic vasospasm and inflammatory reactions in cerebral vessels (3). In a rodent model of SAH Lin et al. assessed the extent of vasospasm by measuring cross-section surfaces of the basilar artery and analyzing the expressions of eNOS and iNOS after treatment with estradiol (15). In the view of Lin et al., the findings indicate vasodilatory effects of estradiol on cerebral arteries by averting an increase of iNOS expression and maintaining regular eNOS levels (15). Zancan et al. demonstrated in smooth muscle cell cultures of rat aorta that estradiol decreased the quantity and activity of iNOS presumably via the estrogen receptor and may also attenuate a provoked iNOS rise (16). Likewise, in a rat model of subarachnoid hemorrhage, Shih et al. showed that the treatment with estradiol inhibited vasospasm as well as elevations of iNOS protein levels (17). In the context of cerebral vasospasm and endothelial damage other studies focused on endothelins (ET) with ET-1 being the isoform exerting strong vasoconstrictive properties $(18,19)$. As ET-1 may foster cerebral vasoconstriction in aSAH, Lin et al. investigated the effect of treatment with estradiol on ET-1 levels and cerebral vasospasm in a SAH rat model (20). Lin et al. found a significant correlation between cross-section surfaces of the basilar artery and concentrations of ET-1 with a decrease of ET-1 levels in the rodents treated with estradiol thus 
suggesting an advantageous impact of estradiol on vasospasm (20). Interestingly, Macia et al. analyzed the temporal relationship between increased ET levels and neurological deterioration in patients with aSAH and, however, found increased ET levels in patients with poor neurological condition apparently independent of the existence of cerebral vasospasm (21).

The neuroprotective influences of progesterone and testosterone have been investigated to a lesser extent. In a rat model with induced SAH Chang et al. showed that both the vasospasm and the lowering of eNOS protein levels in these rodents could be counteracted by therapy with progesterone (5). In a mice SAH model Turan et al. demonstrated that progesterone treatment decreased cerebral vasospasm and had beneficial effects on behavior patterns (22). However, as mentioned above, Zancan et al. demonstrated in cells cultures of rat aorta that estradiol decreased the content of iNOS but such an effect could not be shown with progesterone (16).

The relevance of testosterone to SAH remains rather undetermined as studies are scarce. In rabbits with induced SAH, Gurer et al. evaluated the vasodilatory and neuroprotective properties of testosterone and reasoned that intraperitoneal application of testosterone protected against cerebral vasospasm and neuronal damage (23). Selles et al., however, noted that the enhanced NOS activity in endothelial cells of rat aorta after treatment with estradiol and progesterone could not be shown for testosterone (11).

In the present study, in humans with aSAH, merely very weak correlations were found between transcranial Doppler flow velocities of cerebral arteries and concentrations of estradiol, progesterone and testosterone in serum or CSF. In contrast, the aforementioned studies in rodents with experimental SAH demonstrated beneficial effects of estradiol and progesterone on cerebral vasospasm, often using crosssection surfaces of cerebral arteries as a parameter $(5,15,17,20)$. In the view of these studies, the findings in rodents appear not to be in accordance with the results of our study in humans. However, the comparability of studies using different parameters to evaluate cerebral vasospasm, such as crosssection of arteries and Doppler flow velocities respectively, may be limited. To the best of our knowledge, there are no studies in rodents on flow velocities of cerebral arteries after experimental SAH. In addition, caution is required when comparing the findings in rodents with the findings in humans. Lin et al. also stated in their study in rodents on the attenuation of cerebral vasospasm after experimental SAH by estradiol that their findings in rats may not necessarily be transferred to humans (4). These considerations apply and complicate the interpretation of the results in the present study.

In that regard, the discussion of the results of the present study should include general considerations on steroid metabolism. In the periphery, the steroid hormones progesterone, testosterone and estradiol are synthesized in steroidogenic tissues pursuing effects in the periphery and may also cross the blood-brain barrier and act centrally $(24,25)$. Within the brain, steroids can also be synthesized de novo $(26-28)$ although the mechanisms regulating this synthesis remain mostly unclear $(25,27,29-31)$. In addition, a conversion of steroids by neural cells into metabolites also occurs $(32,33)$. In our previous study on males without neurological disorders or diseases only weak to very weak correlations were found for estradiol, progesterone and testosterone between the CSF and serum compartments thus suggesting that 
concentrations in the periphery do not parallel concentrations in the central compartments (34). Also, the synthesis and conversion of steroids further increases the complexity in which these molecules affect the function of the nervous system and related structures as for example blood vessels. It thus remains unclear, to what extent an administration of exogenous estradiol, as performed in the aforementioned rodent studies, may result in altered estradiol concentrations in the respective compartments, e.g. blood, CSF or brain parenchyma, thereby potentially influencing the course of cerebral vasospasm. Further research is needed to clarify these aspects.

It is understood that a poor neurological condition or a neurological deterioration may be due to cerebral vasospasm but a poor neurological condition can also be attributed to proapoptotic mechanisms unrelated to cerebral vasospasm $(2,35)$. Studies in rodents suggest that steroids may have favorable influences on apoptotic pathways, there among studies in rodents with traumatic and ischemic brain injury (36-39) as well as studies in rodents with experimental SAH $(4,40-43)$. However, in the present study, with regard to possible general neuroprotective effects of steroids, the group comparisons between steroid concentrations in the respective compartments and the grading of the Hunt \& Hess scale, the grading of the Glascow Outcome scale and the presence of cerebral infarction revealed no significant differences. Interestingly, in our previous research evaluating cognitive performance and histological damage in rats with experimental SAH no differences were found between rodents with different hormonal status (44).

The strength of the present study is its clinical approach with a study cohort consisting of humans with aSAH as rodent models of experimental SAH may not reliably represent the pathophysiological processes of spontaneous aSAH. To the best of our knowledge, there are no investigations in humans with aSAH on correlations between transcranial Doppler flow velocities of cerebral arteries and concentrations of steroids in blood or CSF. In the present study, analyses of estradiol, progesterone and testosterone were performed in both CSF and blood thus not relying solely on measurements in the blood compartment which may not adequately describe concentrations in the CSF (34). A further strength lies in the multiple measurement points after the initial bleeding resulting in approximately 280 observations for the calculation of each correlation.

Limitations of the present study must be considered. Measurements of steroid levels have long been a matter of debate and various methods are described in the literature (45), e.g. radioimmunoassay, enzyme immunoassay, liquid chromatography, gas chromatography-mass spectrometry, competitive protein binding assay. The electro-chemiluminescence immunoassay used in the present study was also used by Schonknecht et al. for the measurement of estradiol levels in CSF of humans with Alzheimer's disease (46) as well as in our previous study on males without neurological disorders for the measurement of estradiol, progesterone and testosterone in CSF and serum (34). Steroid concentrations in CSF were also determined with an enzyme immunoassay by Kawass et al. and Brundu et al. $(47,48)$. Still, methodological deficiencies cannot be excluded. Another limitation is that steroid concentrations representing the central compartment were measured in the CSF obtained via ventricular drainage; thus, these measurements are coupled with the uncertainty that they adequately reflect the respective brain 
steroid concentrations. Consequently, the findings of this study regarding correlations between flow velocities of cerebral arteries and central steroid concentrations rest on concentrations in the CSF but not on brain parenchyma levels.

\section{Conclusions}

In humans with aSAH, the present study demonstrated very weak correlations between transcranial Doppler flow velocities of cerebral arteries and concentrations of estradiol, progesterone and testosterone in CSF and serum. However, a careful interpretation is necessary, and these findings may not preclude the beneficial effects of steroids on cerebral vasospasm that have been suggested in rodent studies. Cerebral vasospasm is a distinct finding in aSAH and may entail neurological deficits but neurological deterioration in the absence of cerebral vasospasm is also widely recognized and pathways may be interrelated. Thus, the idea that steroids may have an influence on cerebral vasospasm or other processes of neurological deterioration remains to be further investigated.

\section{Abbreviations}

aSAH: aneurysmal subarachnoid hemorrhage; CSF: cerebrospinal fluid; SAH: subarachnoid hemorrhage; TCD: transcranial Doppler sonography

\section{Declarations}

\section{Ethics approval and consent to participate}

The study was carried out according to the Declaration of Helsinki and the Guideline for Good Clinical Practice. The study was approved by the ethics committee of the medical faculty of the Technical University Munich (2410/09). All participants or their legal guardians signed an informed consent.

\section{Consent for publication}

Not applicable.

\section{Availability of data and materials}

The datasets used and/or analyzed during the current study are available from the corresponding author on reasonable request.

\section{Competing interests}

The authors declare that they have no competing interests. 


\section{Funding}

Not applicable.

\section{Authors' contributions}

JM designed the study, analyzed and interpreted the data and drafted the manuscript. EP acquired and interpreted the data. BU analyzed and interpreted the data. JG interpreted the data and revised the manuscript. MW interpreted the data and revised the manuscript. BJ analyzed and interpreted the data and revised the manuscript. SK designed the study, analyzed and interpreted the data and revised the manuscript. All authors read and approved the final manuscript.

\section{Acknowledgements}

Not applicable.

\section{References}

1. Macdonald RL. Pathophysiology and molecular genetics of vasospasm. Acta Neurochir Suppl. 2001;77:7-11.

2. Ding D, Starke RM, Dumont AS, Owens GK, Hasan DM, Chalouhi N, et al. Therapeutic implications of estrogen for cerebral vasospasm and delayed cerebral ischemia induced by aneurysmal subarachnoid hemorrhage. Biomed Res Int. 2014;2014:727428.

3. Handa Y, Kabuto M, Kobayashi H, Kawano H, Takeuchi H, Hayashi M. The correlation between immunological reaction in the arterial wall and the time course of the development of cerebral vasospasm in a primate model. Neurosurgery. 1991;28(4):542-9.

4. Lin CL, Dumont AS, Su YF, Tsai YJ, Huang JH, Chang KP, et al. Attenuation of cerebral vasospasm and secondary injury by 17 beta-estradiol following experimental subarachnoid hemorrhage. $J$ Neurosurg. 2009;110(3):457-61.

5. Chang CM, Su YF, Chang CZ, Chung CL, Tsai YJ, Loh JK, et al. Progesterone attenuates experimental subarachnoid hemorrhage-induced vasospasm by upregulation of endothelial nitric oxide synthase via Akt signaling pathway. Biomed Res Int. 2014;2014:207616.

6. Hunt WE, Hess RM. Surgical risk as related to time of intervention in the repair of intracranial aneurysms. J Neurosurg. 1968;28(1):14-20.

7. Jennett B, Bond M. Assessment of outcome after severe brain damage. Lancet. 1975;1(7905):480-4.

8. Knowles RG, Moncada S. Nitric oxide synthases in mammals. Biochem J. 1994;298(Pt 2):249-58.

9. Chen Z, Yuhanna IS, Galcheva-Gargova Z, Karas RH, Mendelsohn ME, Shaul PW. Estrogen receptor alpha mediates the nongenomic activation of endothelial nitric oxide synthase by estrogen. $\mathrm{J}$ Clin 
Invest. 1999;103(3):401-6.

10. Nevzati E, Shafighi M, Bakhtian KD, Treiber H, Fandino J, Fathi AR. Estrogen induces nitric oxide production via nitric oxide synthase activation in endothelial cells. Acta Neurochir Suppl. 2015;120:141-5.

11. Selles J, Polini N, Alvarez C, Massheimer V. Progesterone and 17 beta-estradiol acutely stimulate nitric oxide synthase activity in rat aorta and inhibit platelet aggregation. Life Sci. 2001;69(7):81527.

12. Khurana VG, Smith LA, Baker TA, Eguchi D, O'Brien T, Katusic ZS. Protective vasomotor effects of in vivo recombinant endothelial nitric oxide synthase gene expression in a canine model of cerebral vasospasm. Stroke. 2002;33(3):782-9.

13. Link TE, Murakami K, Beem-Miller M, Tranmer BI, Wellman GC. Oxyhemoglobin-induced expression of R-type Ca2 + channels in cerebral arteries. Stroke. 2008;39(7):2122-8.

14. Macdonald RL, Weir BK. Cerebral vasospasm and free radicals. Free Radic Biol Med. 1994;16(5):633-43.

15. Lin CL, Shih HC, Dumont AS, Kassell NF, Lieu AS, Su YF, et al. The effect of 17beta-estradiol in attenuating experimental subarachnoid hemorrhage-induced cerebral vasospasm. J Neurosurg. 2006;104(2):298-304.

16. Zancan V, Santagati S, Bolego C, Vegeto E, Maggi A, Puglisi L. 17Beta-estradiol decreases nitric oxide synthase II synthesis in vascular smooth muscle cells. Endocrinology. 1999;140(5):2004-9.

17. Shih HC, Lin CL, Lee TY, Lee WS, Hsu C. 17beta-Estradiol inhibits subarachnoid hemorrhage-induced inducible nitric oxide synthase gene expression by interfering with the nuclear factor kappa B transactivation. Stroke. 2006;37(12):3025-31.

18. Davenport AP, Hyndman KA, Dhaun N, Southan C, Kohan DE, Pollock JS, et al. Endothelin Pharmacol Rev. 2016;68(2):357-418.

19. Miyauchi T, Masaki T. Pathophysiology of endothelin in the cardiovascular system. Annu Rev Physiol. 1999;61:391-415.

20. Lin CL, Dumont AS, Wu SC, Wang CJ, Howng SL, Huang YF, et al. 17beta-estradiol inhibits endothelin1 production and attenuates cerebral vasospasm after experimental subarachnoid hemorrhage. Exp Biol Med (Maywood). 2006;231(6):1054-7.

21. Mascia L, Fedorko L, Stewart DJ, Mohamed F, terBrugge K, Ranieri VM, et al. Temporal relationship between endothelin-1 concentrations and cerebral vasospasm in patients with aneurysmal subarachnoid hemorrhage. Stroke. 2001;32(5):1185-90.

22. Turan N, Miller BA, Huie JR, Heider RA, Wang J, Wali B, et al. Effect of Progesterone on Cerebral Vasospasm and Neurobehavioral Outcomes in a Rodent Model of Subarachnoid Hemorrhage. World Neurosurg. 2018;110:e150-e9.

23. Gurer B, Turkoglu E, Kertmen H, Karavelioglu E, Arikok AT, Sekerci Z. Attenuation of cerebral vasospasm and secondary injury by testosterone following experimental subarachnoid hemorrhage in rabbit. Acta Neurochir (Wien). 2014;156(11):2111-20. discussion 20. 
24. Hobbs CJ, Jones RE, Plymate SR. The effects of sex hormone binding globulin (SHBG) on testosterone transport into the cerebrospinal fluid. J Steroid Biochem Mol Biol. 1992;42(6):629-35.

25. Tsutsui K, Ukena K, Usui M, Sakamoto $H$, Takase M. Novel brain function: biosynthesis and actions of neurosteroids in neurons. Neurosci Res. 2000;36(4):261-73.

26. Baulieu EE. Neurosteroids: a novel function of the brain. Psychoneuroendocrinology. 1998;23(8):963-87.

27. Porcu P, Barron AM, Frye CA, Walf AA, Yang SY, He XY, et al. Neurosteroidogenesis Today: Novel Targets for Neuroactive Steroid Synthesis and Action and Their Relevance for Translational Research. J Neuroendocrinol. 2016;28(2):12351.

28. Robel P, Baulieu EE. Neurosteroids Biosynthesis and function. Trends Endocrinol Metab. 1994;5(1):1-8.

29. Agis-Balboa RC, Pinna G, Zhubi A, Maloku E, Veldic M, Costa E, et al. Characterization of brain neurons that express enzymes mediating neurosteroid biosynthesis. Proc Natl Acad Sci U S A. 2006;103(39):14602-7.

30. Do Rego JL, Seong JY, Burel D, Luu-The V, Larhammar D, Tsutsui K, et al. Steroid biosynthesis within the frog brain: a model of neuroendocrine regulation. Ann N Y Acad Sci. 2009;1163:83-92.

31. Charlier TD, Cornil CA, Patte-Mensah C, Meyer L, Mensah-Nyagan AG, Balthazart J. Local modulation of steroid action: rapid control of enzymatic activity. Front Neurosci. 2015;9:83.

32. Melcangi RC, Garcia-Segura LM, Mensah-Nyagan AG. Neuroactive steroids: state of the art and new perspectives. Cell Mol Life Sci. 2008;65(5):777-97.

33. Rose KA, Stapleton G, Dott K, Kieny MP, Best R, Schwarz M, et al. Cyp7b, a novel brain cytochrome $\mathrm{P} 450$, catalyzes the synthesis of neurosteroids 7alpha-hydroxy dehydroepiandrosterone and 7alphahydroxy pregnenolone. Proc Natl Acad Sci U S A. 1997;94(10):4925-30.

34. Martin J, Plank E, Jungwirth B, Hapfelmeier A, Podtschaske A, Kagerbauer SM. Weak correlations between serum and cerebrospinal fluid levels of estradiol, progesterone and testosterone in males. BMC Neurosci. 2019;20(1):53.

35. Cahill J, Calvert JW, Zhang JH. Mechanisms of early brain injury after subarachnoid hemorrhage. J Cereb Blood Flow Metab. 2006;26(11):1341-53.

36. Liu R, Wang X, Liu Q, Yang SH, Simpkins JW. Dose dependence and therapeutic window for the neuroprotective effects of 17 beta-estradiol when administered after cerebral ischemia. Neurosci Lett. 2007;415(3):237-41.

37. Wen Y, Yang S, Liu R, Perez E, Yi KD, Koulen P, et al. Estrogen attenuates nuclear factor-kappa B activation induced by transient cerebral ischemia. Brain Res. 2004;1008(2):147-54.

38. Chiappetta O, Gliozzi M, Siviglia E, Amantea D, Morrone LA, Berliocchi L, et al. Evidence to implicate early modulation of interleukin-1beta expression in the neuroprotection afforded by 17beta-estradiol in male rats undergone transient middle cerebral artery occlusion. Int Rev Neurobiol. 2007;82:35772. 
39. O'Connor CA, Cernak I, Johnson F, Vink R. Effects of progesterone on neurologic and morphologic outcome following diffuse traumatic brain injury in rats. Exp Neurol. 2007;205(1):145-53.

40. Kao CH, Chang CZ, Su YF, Tsai YJ, Chang KP, Lin TK, et al. 17beta-Estradiol attenuates secondary injury through activation of Akt signaling via estrogen receptor alpha in rat brain following subarachnoid hemorrhage. J Surg Res. 2013;183(1):e23-30.

41. Lin CL, Dumont AS, Tsai YJ, Huang JH, Chang KP, Kwan AL, et al. 17Beta-estradiol activates adenosine A(2a) receptor after subarachnoid hemorrhage. J Surg Res. 2009;157(2):208-15.

42. Yan F, Hu Q, Chen J, Wu C, Gu C, Chen G. Progesterone attenuates early brain injury after subarachnoid hemorrhage in rats. Neurosci Lett. 2013;543:163-7.

43. Wang Z, Zuo G, Shi XY, Zhang J, Fang Q, Chen G. Progesterone administration modulates cortical TLR4/NF-kappaB signaling pathway after subarachnoid hemorrhage in male rats. Mediators Inflamm. 2011;2011:848309.

44. Kagerbauer SM, Kadera V, Gordan LM, Blobner M, Torok E, Schmid S, et al. Influence of sex and hormonal status on initial impact and neurocognitive outcome after subarachnoid haemorrhage in rats. Behav Brain Res. 2019;363:13-22.

45. Kancheva R, Hill M, Novak Z, Chrastina J, Velikova M, Kancheva L, et al. Peripheral neuroactive steroids may be as good as the steroids in the cerebrospinal fluid for the diagnostics of CNS disturbances. J Steroid Biochem Mol Biol. 2010;119(1-2):35-44.

46. Schonknecht P, Henze M, Hunt A, Klinga K, Haberkorn U, Schroder J. Hippocampal glucose metabolism is associated with cerebrospinal fluid estrogen levels in postmenopausal women with Alzheimer's disease. Psychiatry Res. 2003;124(2):125-7.

47. Brundu B, Loucks TL, Adler LJ, Cameron JL, Berga SL. Increased cortisol in the cerebrospinal fluid of women with functional hypothalamic amenorrhea. J Clin Endocrinol Metab. 2006;91(4):1561-5.

48. Kawwass JF, Sanders KM, Loucks TL, Rohan LC, Berga SL. Increased cerebrospinal fluid levels of GABA, testosterone and estradiol in women with polycystic ovary syndrome. Hum Reprod. 2017;32(7):1450-6.

\section{Tables}

Due to technical limitations, table 1, table 2 , table 3 and table 4 are only available as a download in the Supplemental Files section.

\section{Supplementary Files}

This is a list of supplementary files associated with this preprint. Click to download.

- supplement1.xlsx

- supplement2.xlsx

- Table1Patientcharacteristics.xlsx 
- supplement4.xlsx

Page 13/13 